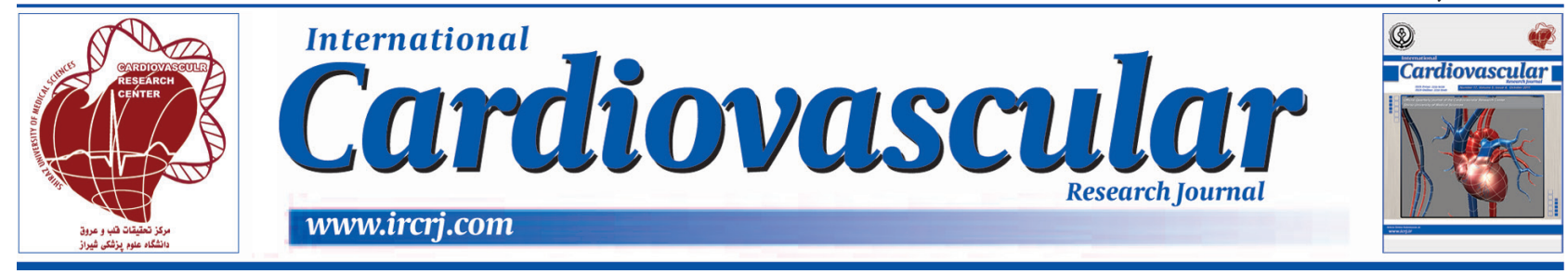

\title{
Right Ventricular Involvement in either Anterior or Inferior Myocardial Infarction
}

\author{
Firoozeh Abtahi ${ }^{1}$, Mahkameh Farmanesh ${ }^{1}$, Alireza Moaref ${ }^{1}$, Shahnaz Shekarforoush 2,*
}

${ }^{1}$ Cardiovascular Research Center, Shiraz University of Medical Sciences, Shiraz, IR Iran

${ }^{2}$ Department of Physiology, Arsanjan Branch, Islamic Azad University, Arsanjan, IR Iran

\begin{tabular}{l}
\hline A R T I C L E I N F O \\
\hline Article Type: \\
Research Article \\
\hline Article History: \\
Received: 21 Apr 2015 \\
Revised: 31 Aug 2015 \\
Accepted: 28 Sep 2015
\end{tabular}

\section{Keywords:}

Ventricular Dysfunction

Echocardiography

Myocardial Infarction

\begin{abstract}
A B S T R A C T
Background: Unlike left ventricular function, less attention has been paid to Right Ventricular (RV) function after Myocardial Infarction (MI).

Objectives: The current study aimed to compare RV function in patients with inferior and anterior MI.

Patients and Methods: During the study period, 60 patients consecutively presented to the Emergency Department with chest pain were divided into two groups based on their electrocardiographic findings. Accordingly, 25 patients had inferior MI (IMI group) and 35 ones had anterior MI (AMI group). Echocardiography was performed 48 hours after starting the standard therapy. Conventional echocardiographic parameters and Tissue Doppler Imaging (TDI) measurements were acquired from the standard views. Student t-test and the chi-square test were respectively used for comparisons of the normally distributed continuous and categorical variables in the two groups. Besides, $\mathrm{P}<0.05$ was considered to be statistically significant.
\end{abstract}

Results: The ratio of early diastolic velocity of the tricuspid flow to early diastolic velocity of the tricuspid annulus in the lateral side (E/E') was significantly higher in the AMI group compared to the IMI group $(6.73 \pm 1.64$ vs. $5.68 \pm 1.32, \mathrm{P}=0.01)$. In addition, abnormal E/E' ratio of the tricuspid valve was present in 27 (77\%) and 14 (56\%) patients in the anterior and inferior MI groups, respectively. Besides, the RV Tei index was significantly higher in the AMI group compared to the IMI group $(0.65 \pm 0.11$ vs. $0.58 \pm$ $0.13, \mathrm{P}=0.02)$. Overall, 28 patients $(80 \%)$ with anterior infarction and 12 patients $(48 \%)$ with inferior infarction had abnormal RV Tei index. However, no significant difference was found between the two groups regarding other variables.

Conclusions: RV dysfunction commonly occurs after a first acute ST-elevation MI. However, RV functional changes are more pronounced in patients with anterior MI.

Implication for health policy/practice/research/medical education:

This study showed that right ventricular dysfunction commonly occurs after a first acute ST-elevation myocardial infarction. However, right ventricular functional changes are more pronounced in patients with anterior MI.

\section{Background}

Assessment of Left Ventricular (LV) function using 2D echocardiography shortly after acute Myocardial Infarction (MI) is essential and one of the most important prognostic parameters. However, the association between Right Ventricular (RV) function and adverse events after acute

*Corresponding author: Shahnaz Shekarforoush, Arsanjan Branch, Islamic Azad University, Arsanjan, Iran. Cellphone: +98-9173376859, Fax: +98-7297622483,

E-mail:sh.shekar@yahoo.com
MI is poorly known, especially in patients with mild LV dysfunction (1).

Because of therapeutic implications, there has been growing interest in early recognition of RV infarction with non-invasive techniques. Zornoff et al. demonstrated that in patients with Left Ventricular Ejection Fraction (LVEF) $\leq$ $40 \%$, RV function was a significant independent predictor of death and development of heart failure after an acute MI (2). Thus, quantitative assessment of RV function after MI should be noted. 
Up to now, most studies on RV function after MI have concentrated on patients with inferior infarction. Nearly $50 \%$ of patients with inferior infarcts and $10 \%$ or fewer patients with anterior infarcts show evidence of RV involvement $(3,4)$. Yet, inferior and anterior infarctions lead to quite different RV hemodynamic responses. Patients with inferior infarction, despite equivalent infarct size, are associated with a much lower mortality rate compared to those with anterior infarction. Therefore, the site of MI can predict the severity and extension of infarction. Furthermore, some investigators have reported RV dysfunction after inferior infarction, but not after anterior infarction (5), while others have found it in both groups (6).

\section{Objectives}

Due to these conflicting reports, the current study aims to compare the extent of RV dysfunction in patients admitted during the first acute ST-segment elevation inferior or anterior infarction.

\section{Patients and Methods}

\subsection{Patient Selection}

This study was conducted on 60 patients who had referred to the Emergency Department of Shahid Faghihi hospital (in Shiraz) with chest pain and were hospitalized with a diagnosis of acute MI for the first time from February to September 2013.

The subjects were divided into two groups based on Electrocardiographic (ECG) findings. Group I (Inferior Myocardial Infarction, IMI) consisted of 25 patients with ST-elevation in the inferior and posterior leads (II, III, aVF, V7, and V8) and group II (Anterior Myocardial Infarction, AMI) included 35 patients with ST-elevation in the precordial and lateral leads (V1-V6, aVL, and I). An echocardiography was performed 48 hours after the beginning of standard treatment, including Percutaneous Coronary Intervention (PCI) or fibrinolysis. Out of the 60 patients who met the inclusion criteria, 21 were treated by primary PCI (10 and 11 with inferior and anterior MI, respectively) and 39 were treated with streptokinase (15 and 24 with inferior and anterior MI, respectively).

This study was approved by the Review Board and Ethics Committee of Shiraz University of Medical Sciences. Written informed consents were also obtained from all the patients. The patients who had bundle branch block or any other intraventricular conduction delay and previous history of MI, valvular disease, heart failure, and pulmonary hypertension were excluded from the study.

\subsection{Echocardiographic Evaluations}

Standard 2-D transthoracic echocardiography and Tissue
Doppler Imaging (TDI) were performed for the patients in the left lateral decubitus position by Vivid 9 GE ultrasound system (Horten, Norway) according to the recommendation of the American Society of Echocardiography. Besides, LVEF was calculated using the biplane Simpson method (7). We sought to investigate the association between LVEF and other measured parameters.

Apical four-chamber view was used to obtain the conventional echocardiographic parameters and TDI measurements. RV minor dimension at the mid-ventricular level was measured in end-diastole. Care had to be taken to obtain a true non-foreshortened apical four-chamber view to achieve the maximum RV dimension. Right Atrial (RA) minor dimension was also measured in end-systole.

Tricuspid Annular Plane Systolic Excursion (TAPSE) was measured as an index of the global systolic function by placing $\mathrm{M}$-mode cursor along the tricuspid valve annulus in apical four-chamber view. Moreover, pulsed Doppler RV diastolic indexes were measured in apical fourchamber view, placing the sample volume at the tip of the tricuspid valve. E and A velocities ( $\mathrm{m} / \mathrm{s})$ and their ratio were determined, as well.

The myocardial performance index (Tei index), as a simple and reproducible index of combined evaluation of systolic and diastolic function that was first measured by Tei et al. in 1995, was calculated as: (IVRT + IVCT)/ET (8) (Figure 1).

\subsection{Statistical Analysis}

The statistical analyses were performed using the SPSS statistical software (version 16.0, SPSS Inc., Chicago, IL, USA). First, the data were assessed for normality using Kolmogorov-Smirnov test. Then, continuous data were presented as mean \pm Standard Deviation (SD) and categorical data were presented as frequencies and percentages. Student t-test and chi-square test were respectively used for comparison of the normally distributed continuous and categorical variables in the two groups. Pearson's correlation coefficient was also employed to determine the strength of linear relationships between the study variables and LVEF. P value $<0.05$ was considered to be statistically significant.

\section{Results}

This study was conducted on 60 patients, 25 in group 1 (IMI) and 35 in group 2 (AMI). The baseline characteristics of the patients have been listed in Table 1 .

The indices measured by echocardiography have been presented in Table 2. Accordingly, LVEF was lower in the patients with anterior infarction, but the difference was not statistically significant. Also, no significant difference was observed between the patients with AMI and IMI regarding

\begin{tabular}{llll}
\hline Table 1. The Patients' Baseline Characteristics and Hemodynamic Values & & \\
\hline & Inferior $(\mathbf{n}=\mathbf{2 5})$ & Anterior $(\mathbf{n}=\mathbf{3 5})$ \\
\hline Age (years) & $60 \pm 10.2$ & $59 \pm 12.9$ & \\
Female/male & $12 / 13$ & $12 / 23$ & \\
Blood pressure (mmHg) & & & \\
Systolic & $118 \pm 7$ & $117 \pm 9$ & \\
Diastolic & $74 \pm 6$ & $73 \pm 6$ & 0.3 \\
Heart rate (beats/min) & $80 \pm 7$ & $78 \pm 7$ & 0.59 \\
\hline
\end{tabular}

The results have been expressed as mean \pm SD. 
Figure 1. Calculation of Right Ventricular Myocardial Performance Index (Tei Index). Time Intervals Measured from Pulsed Tissue Doppler Were Obtained from the Lateral Tricuspid Annulus
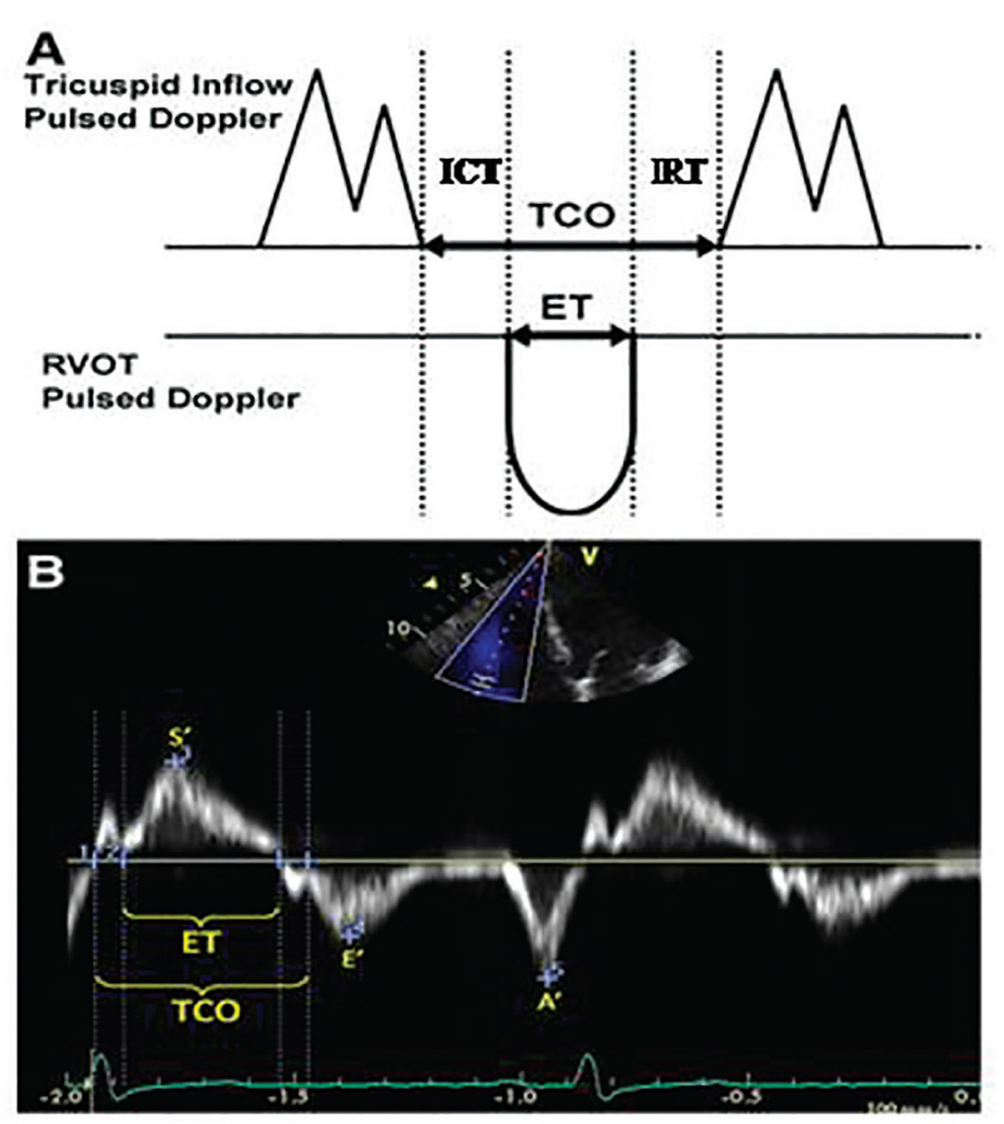

Abbreviations: $\mathrm{TCO}=$ from the onset of isovolumic contraction to the onset of diastolic filling and ET $=$ right ventricular ejection time. The sum of Isovolumic Contraction Time (ICT) and Isovolemic Relaxation Time (IRT) can be obtained by subtracting ET from TCO; MPI $=(\mathrm{TCO}-\mathrm{ET}) / \mathrm{ET}$.

\begin{tabular}{llll}
\hline \multicolumn{2}{l}{ Table 2. Comparison of the Patients with Anterior and Inferior Infarction Regarding Right Ventricular Functions } \\
\hline & Inferior $(\mathbf{n}=\mathbf{2 5})$ & Anterior $(\mathbf{n}=\mathbf{3 5})$ & P value \\
\hline RV end-diastolic diameter $(\mathbf{m m})$ & $29 \pm 4$ & $30.3 \pm 4$ & 0.30 \\
RA size of minor axis $(\mathbf{c m})$ & $3.3 \pm 0.5$ & $3.3 \pm 0.3$ & 0.8 \\
IVC size (cm) & $1.5 \pm 0.3$ & $1.5 \pm 0.3$ & 0.7 \\
E/A ratio of tricuspid & $1.24 \pm 0.44$ & $1.23 \pm 0.55$ & 0.88 \\
E/E' ratio of tricuspid & $5.68 \pm 1.32$ & $6.73 \pm 1.64$ & 0.01 \\
E' of annulus of tricuspid (cm/s) & $7.76 \pm 1.78$ & $7.54 \pm 2.22$ & 0.68 \\
Tricuspid annular systolic excursion, TAPSE $(\mathbf{m m})$ & $17.4 \pm 2$ & $17.3 \pm 1.7$ & 0.90 \\
Lateral tricuspid annular systolic velocity, S' (cm/s) & $9.48 \pm 1.66$ & $9.54 \pm 2.74$ & 0.91 \\
RV Tei index & $0.58 \pm 0.13$ & $0.65 \pm 0.11$ & 0.02 \\
VTI S' lateral tricuspid annular systolic wave & $1.78 \pm 0.39$ & $1.65 \pm 0.44$ & 0.24 \\
LVEF (\%) & $41 \pm 8$ & $37 \pm 6$ & 0.10 \\
\hline
\end{tabular}

Abbreviations: LVEF, left ventricular ejection fraction; RV, right ventricle; RA, right atrium; IVC, inferior vena cava

The data have been presented as mean $\pm \mathrm{SD}$.

RV and RA diameters. RV involvement occurred in up to $50 \%$ of the patients with acute MI.

Considering the parameters of RV function, no significant differences were found between the anterior and inferior infarction groups regarding the measurements of E/A ratio of the tricuspid valve, TAPSE, and S' velocity. However, TDI showed that the patients with anterior infarction had a significantly higher mean $\mathrm{E} / \mathrm{E}$ ' ratio in comparison to those with inferior infarction $(6.73 \pm 1.64$ vs. $5.68 \pm 1.32, \mathrm{p}=0.01)$. $\mathrm{E} / \mathrm{E}^{\prime}>6$ was found in 27 out of the 35 patients with anterior infarction (77\%). The RV Tei index was also significantly higher in the anterior infarction group compared to the inferior infarction group $(\mathrm{P}=0.02)$.

The results showed a significant positive correlation between LVEF and E/E' of tricuspid inflow ( $\mathrm{r}=0.2, \mathrm{P}=$ $0.04)$, lateral tricuspid annular systolic velocity or $S^{\prime}$ ( $r$ $=0.30, \mathrm{P}=0.01)$, VTI S' of the lateral tricuspid annular systolic wave $(r=0.28, P=0.02)$, and TAPSE $(r=0.39, P$ $=0.002$ ). Also, a significant negative correlation was found between EF and Inferior Vena Cava (IVC) size ( $\mathrm{r}=-0.27$, 
$\mathrm{P}=0.03)$

\section{Discussion}

The results of the current study demonstrated that both anterior and inferior infarction had marked effects on RV function. RV systolic and diastolic function indices were clearly impaired in such large number of subjects with MI. Although no significant differences were observed between the two groups regarding the measurements of most RV function indices, E/E' ratio of the tricuspid inflow, as an index of RV diastolic function, and RV Tei index by TDI, as a measure of global RV performance, were significantly higher in the patients with anterior infarction compared to those with inferior infarction. The study also revealed that LVEF decline, irrespective of the site of MI, was strongly associated with RV involvement. In agreement with this result, Hsu et al. showed that RV function might be affected discrepantly by the infarction site in patients with a first acute ST-elevation MI without concomitant RV infarction. Additionally, only regional RV diastolic dysfunction was observed in patients with inferior infarction, whereas depressed global RV function was present in those with anterior infarction (9). Jensen et al. also revealed RV involvement in $47 \%$ of patients with inferior infarction and $65 \%$ of those with anterior infarction via cardiac Magnetic Resonance Imaging (MRI) (10).

Considering RV involvement in MI and its high mortality, more attention should be paid to detection of RV function in acute MI (4). The results of a study suggested that RV function provided important information for prognosis after MI in patients treated with primary PCI and relatively preserved LV function (1). Besides, another study on 423 patients with normal LV function revealed that reduced RVEF had a mild relationship with one-year mortality (11). In contrast, in a study on 416 patients with LV dysfunction, $\mathrm{RV}$ function independently predicted cardiovascular mortality (2). These results show that changes in RV function subsequent to $\mathrm{MI}$ are closely related to $\mathrm{LV}$ alterations. However, in a meta-analysis conducted by Mehta et al., RV dysfunction in inferior infarction was not correlated to the extent of LV myocardial damage (11).

In addition to RVEF, RV Tei index and systolic and diastolic tricuspid annular velocities are other indices used in assessment of RV function. RV Tei index is important in assessment of global RV performance and estimates RVEF with good accuracy (11). It usually increases in the presence of RV systolic or diastolic dysfunction (12). A higher value of this index in patients with ST-elevation MI is associated with more adverse outcomes (13). The results given in the present paper correspond to the clinical study by Moller et al., which demonstrated that an abnormal RV Tei index was present in $80 \%$ of patients with MI (13). Ozturk et al. also found a significant increase in this index after anterior ST-elevation MI (14). In that study, the alteration of RV Tei index was more pronounced in patients with anterior MI. Similar to our study, the findings of the study by Hsu et al. revealed that RV Tei index was significantly higher in anterior MI than in inferior MI (9). In the research by Sonmez et al., both systolic and diastolic functions were affected after anterior ST-elevation MI. They also found a significant increase in RV Tei index, as an important factor affecting prognosis, after anterior MI (15).

In the recent studies, the tricuspid $\mathrm{E} / \mathrm{E}$ ' ratio and diastolic strain rate have been commonly used to evaluate RV diastolic function. These studies indicated a strong relationship between the tricuspid $\mathrm{E} / \mathrm{E}$ ' ratio and $\mathrm{RA}$ volume, and hemodynamic parameters (16). The value of the tricuspid $\mathrm{E} / \mathrm{E}^{\prime}$ ratio, as a reliable estimate of $\mathrm{RV}$ filling pressure, predicts cardiac events and elevated mean arterial pressure (17). An E/E' ratio greater than 6 had a high sensitivity and specificity in detecting the mean RA pressure $\geq 10 \mathrm{~mm} \mathrm{Hg}$ (18). The results of the current study showed $\mathrm{E} / \mathrm{E}^{\prime}>6$ in $77 \%$ and $56 \%$ of the patients with anterior and inferior MI, respectively. Additionally, the E/E' ratio was significantly higher in anterior infarction. In the research by Hsu et al., E/E' ratio was comparable between anterior and inferior infarction, whereas it was higher in anterior infarction compared to the controls (9). Akdemir et al. also demonstrated that TAPSE, as an indicator of RV function, was lower in patients with acute anterior MI than in the control group in the absence of apparent systolic dysfunction. This was attributed to RV diastolic dysfunction (17).

In conclusion, the present study suggested that RV function was extremely affected in the patients with a first acute ST-elevation MI. Besides, RV involvement was more pronounced in anterior MI than in inferior MI. Moreover, LVEF decline, irrespective of the site of infarction, affected RV function.

\section{Acknowledgements}

The present article was extracted from the thesis written by Dr. Farmanesh and financially supported by Shiraz University of Medical Sciences.

\section{Authors' Contribution}

Study concept and design: Firoozeh Abtahi, Acquisition of data: Mahkameh Farmanesh, Analysis and interpretation of data: Alireza Moaref and Shahnaz Shekarforoush, Drafting of the manuscript: Firoozeh Abtahi and Shahnaz Shekarforoush, Critical revision of the manuscript for important intellectual content: Firoozeh Abtahi and Shahnaz Shekarforoush, Statistical analysis: Shahnaz Shekarforoush, Administrative, technical, and material support: Alireza Moaref, Study supervision: Firoozeh Abtahi

\section{Financial disclosure}

There is no financial disclosure.

\section{Funding/Support}

The study article was financially supported by Shiraz University of Medical Sciences.

\section{References}

1. Antoni ML, Scherptong RW, Atary JZ, Boersma E, Holman ER, van der Wall EE, et al. Prognostic value of right ventricular function in patients after acute myocardial infarction treated with primary percutaneous coronary intervention. Circ Cardiovasc Imaging. 2010;3(3):264-71.

2. Zornoff LA, Skali H, Pfeffer MA, St John Sutton M, Rouleau JL, Lamas GA, et al. Right ventricular dysfunction and risk of heart failure and mortality after myocardial infarction. $\mathrm{J} \mathrm{Am} \mathrm{Coll} \mathrm{Cardiol.}$ 
2002;39(9):1450-5.

3. Gandy WE. Recognition and treatment of right ventricular myocardial infarction. EMS Mag. 2008;37(3):69-73, 100.

4. Pfisterer M. Right ventricular involvement in myocardial infarction and cardiogenic shock. Lancet. 2003;362(9381):392-4.

5. Tobinick E, Schelbert HR, Henning H, LeWinter M, Taylor A, Ashburn WL, et al. Right ventricular ejection fraction in patients with acute anterior and inferior myocardial infarction assessed by radionuclide angiography. Circulation. 1978;57(6):1078-84.

6. Marmor A, Geltman EM, Biello DR, Sobel BE, Siegel BA, Roberts R. Functional response of the right ventricle to myocardial infarction: dependence of the site of left ventricular infarction. Circulation. 1981;64(5):1005-11.

7. Lang RM, Bierig M, Devereux RB, Flachskampf FA, Foster E, Pellikka PA, et al. Recommendations for chamber quantification: a report from the American Society of Echocardiography's Guidelines and Standards Committee and the Chamber Quantification Writing Group, developed in conjunction with the European Association of Echocardiography, a branch of the European Society of Cardiology. $J$ Am Soc Echocardiogr. 2005;18(12):1440-63.

8. Tei C, Ling LH, Hodge DO, Bailey KR, Oh JK, Rodeheffer RJ, et al. New index of combined systolic and diastolic myocardial performance: a simple and reproducible measure of cardiac function--a study in normals and dilated cardiomyopathy. J Cardiol. 1995;26(6):357-66.

9. Hsu SY, Lin JF, Chang SH. Right ventricular function in patients with different infarction sites after a first acute myocardial infarction. Am J Med Sci. 2011;342(6):474-9.

10. Jensen CJ, Jochims M, Hunold P, Sabin GV, Schlosser T, Bruder O. Right ventricular involvement in acute left ventricular myocardial infarction: prognostic implications of MRI findings. AJR Am J
Roentgenol. 2010;194(3):592-8.

11. Salehian O, Schwerzmann M, Merchant N, Webb GD, Siu SC, Therrien J. Assessment of systemic right ventricular function in patients with transposition of the great arteries using the myocardial performance index: comparison with cardiac magnetic resonance imaging. Circulation. 2004;110(20):3229-33.

12. Haddad F, Hunt SA, Rosenthal DN, Murphy DJ. Right ventricular function in cardiovascular disease, part I: Anatomy, physiology, aging, and functional assessment of the right ventricle. Circulation. 2008;117(11):1436-48.

13. Moller JE, Sondergaard E, Poulsen SH, Appleton CP, Egstrup K. Serial Doppler echocardiographic assessment of left and right ventricular performance after a first myocardial infarction. $J \mathrm{Am}$ Soc Echocardiogr. 2001;14(4):249-55.

14. Ozturk O, Ulgen MS, Tekes S, Ozturk U, Toprak N. Influence of angiotensin-converting enzyme I/D gene polymorphism on the right ventricular myocardial performance index in patients with a first acute anterior myocardial infarction. Circ J. 2005;69(2):211-5.

15. Sonmez O, Kayrak M, Altunbas G, Abdulhalikov T, Alihanoglu $\mathrm{Y}$, Bacaksiz A, et al. Right ventricular involvement in anterior myocardial infarction: a tissue Doppler-derived strain and strain rate study. Clinics (Sao Paulo). 2013;68(9):1225-30.

16. Demirkol S, Balta S, Cakar M. Right ventricular diastolic function in patients with thalassemia major. Arq Bras Cardiol. 2013;101(1):93-4.

17. Akdemir O, Yildiz M, Surucu H, Dagdeviren B, Erdogan O, Ozbay G. Right ventricular function in patients with acute anterior myocardial infarction: tissue Doppler echocardiographic approach. Acta Cardiol. 2002;57(6):399-405.

18. Nageh MF, Kopelen HA, Zoghbi WA, Quinones MA, Nagueh SF. Estimation of mean right atrial pressure using tissue Doppler imaging. Am J Cardiol. 1999;84(12):1448-51, A8. 